\title{
Distribution of IgG galactosylation as a promising biomarker for cancer screening in multiple cancer types
}

Cell Research (2016) 26:963-966. doi:10.1038/cr.2016.83; published online 1 July 2016

\section{Dear Editor,}

Cancer is prevalent worldwide. Currently, most routinely used non-invasive serological cancer biomarkers, including carcino-embryonic antigen (CEA), prostate-specific antigen (PSA), $\alpha$-fetoprotein (AFP), CA125, CA15-3, and CA19-9, suffer from low specificity and/ or low sensitivity [1]. Improvements in timely and effective diagnosis of cancer are urgently needed. Most serum proteins are glycosylated, and deregulation of glycosylation has been reported to be associated with a wide range of diseases including cancer [2]. Thus, analysis of blood-based glycans represents a new strategy for cancer diagnosis [2]. Immunoglobin $\mathrm{G}$ ( $\mathrm{IgG}$ ), a highly abundant glycoprotein in serum, is known to mediate a variety of blood immune responses. There has been an increasing interest in the analysis of the $\mathrm{N}$-glycan profile of human IgG in healthy and disease states, such as autoimmune diseases and cancer [3]. Several studies recently showed that the glycosylation profiles of total serum IgG exhibited significant differences between non-malignant controls and cancer patients, such as gastric, liver and ovarian cancers [4-7]. We have previously demonstrated significant decrease in serum level of terminally galactosylated $N$-glycans of IgG in 32 patients with ovarian cancer compared with 26 patients with benign gynecological conditions [7]. Although altered glycosylation of total serum IgG has been profiled individually in ovarian, prostate, gastric, lung, and liver cancers [7, 8], no studies have systematically investigated the alteration of IgG glycosylation in multiple cancers simultaneously. In addition, the sample sizes in previous studies were limited.

In this study, we performed for the first time a largescale, multi-institute study to assess the quantitative changes of IgG glycosylation in 12 types of cancers and non-malignant controls. The primary objective of this study is to investigate whether serum IgG glycosylation patterns have similar alterations in multiple types of cancer and whether alteration in $\operatorname{IgG}$ galactosylation profiles can serve as a pan-cancer biomarker for cancer screening.

To this end, quantitative changes of serum IgG glyco- sylation were assessed by mass spectrometry (MS) based on the method described previously [7]. The detailed methods and statistical analysis were listed in Supplementary information, Data S1. In the discovery stage, we compared the serum IgG glycosylation patterns between healthy individuals and patients with gastric, liver, lung or ovarian cancer. IgG-derived glycans in each sample were assessed by MS, which detected various glycoforms of IgG, such as terminal galactose, core fucose and bisecting N-acetylglucosamine (GlcNAc; Supplementary information, Figure S1A). Comparison of various types of IgG glycosylation between controls and cancer patients showed that only the difference in IgG galactosylation distribution passed the statistical criteria $(P<$ 0.05 ) and achieved a high area under the curve (AUC) score in the receiver operating characteristic (ROC) test (AUC > 0.8) in each cancer type (Supplementary information, Table S1C). Thus, IgG galactosylation was selected as the candidate pan-cancer biomarker for further screening. The distribution of IgG galactosylation (referred to as Gal-ratio) was measured by calculating the relative intensities of agalactosylated (G0) vs monogalactosyl (G1) and digalactosyl (G2) $N$-glycans according to the formula of $\mathrm{G} 0 /(\mathrm{G} 1+\mathrm{G} 2 \times 2)$ [7]. The performance of the IgG Gal-ratio in cancer diagnosis was first evaluated in a small-size test cohort and then validated in an independent large-size cohort.

Participants in the test cohort were recruited from one hospital between September 2013 and April 2014 and include 114 non-cancer controls and 580 cancer patients, covering 12 types of cancers (gastric, liver, lung, ovarian, colorectal, esophageal, pancreatic, renal, prostate, bladder, breast and cervical cancers; 30-50 samples/ cancer type). The Gal-ratios were significantly higher in all cancer patients (median 0.6373, IQR 0.4878-0.8817, mean 0.7445 , SD 0.01745 ) than controls (median 0.3651 , IQR 0.2898-0.4401, mean 0.3841, SD 0.01746; $P<$ 0.0001 ) in the test cohort (Supplementary information, Figure S1B). The validation cohort comprises 5704 participants recruited from three hospitals between September 2013 and March 2016, including 4685 individuals with cancers consisting of the above 12 types, 747 
A
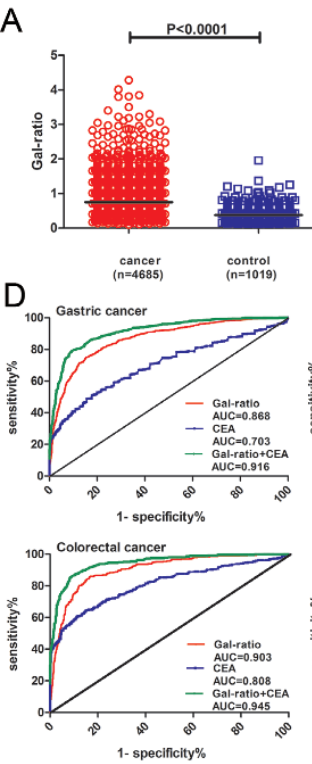

E
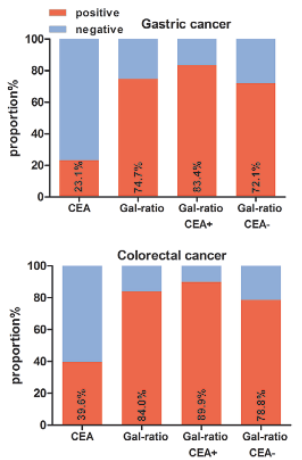

F

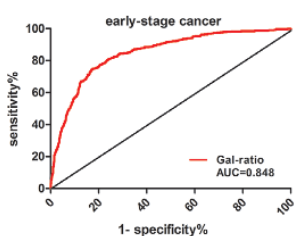

B

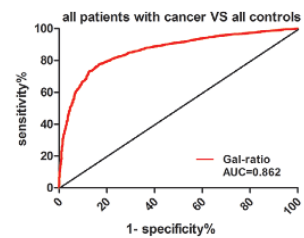

C
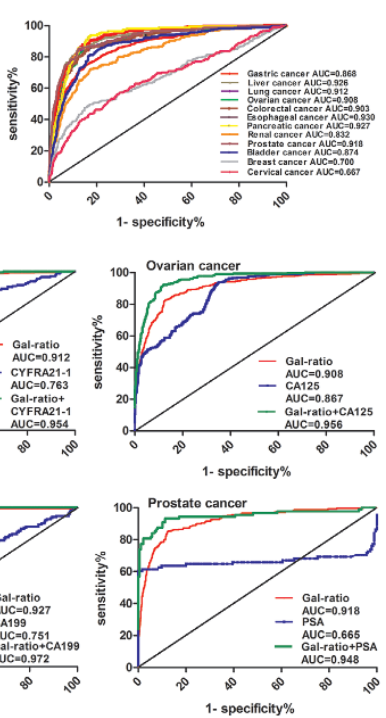
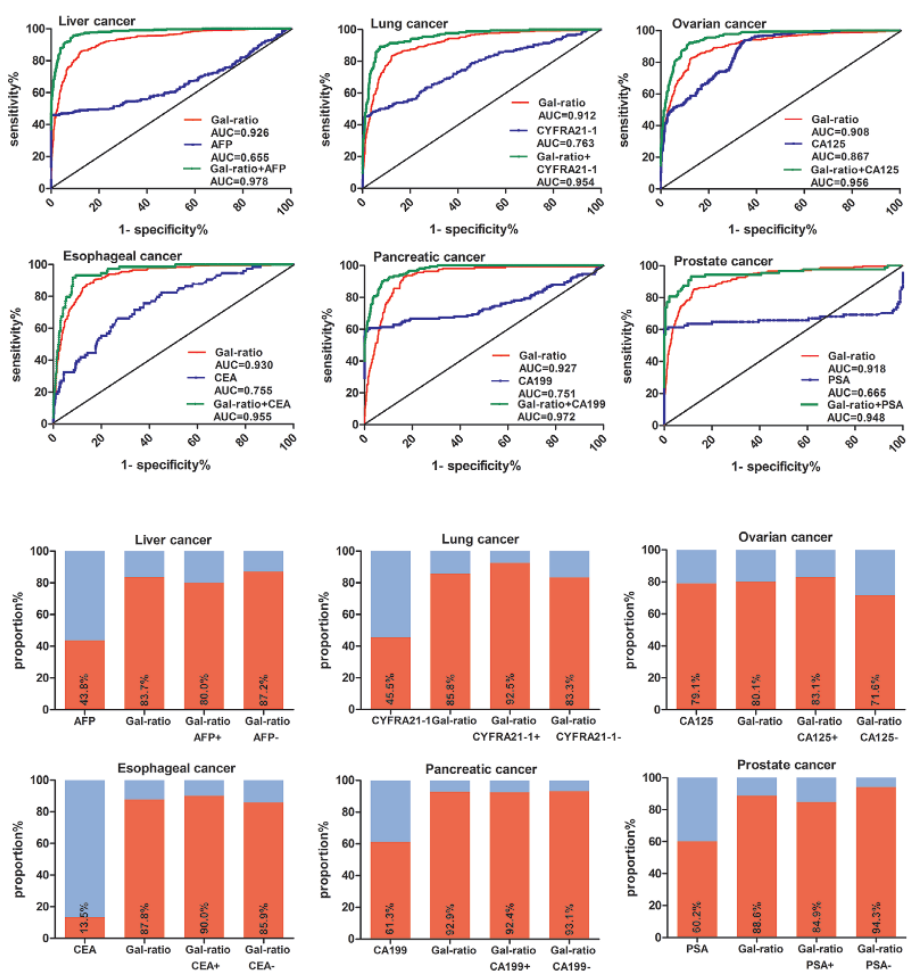

G
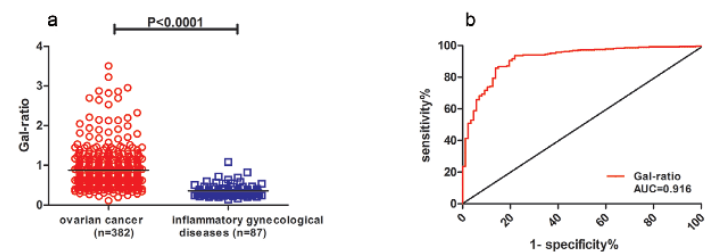

Figure 1 (A) Comparison of IgG Gal-ratio values of serum samples obtained from non-cancer controls and cancer patients in the validation cohort. (B) ROC curve for IgG Gal-ratio to distinguish cancer patients from non-cancer controls in the validation cohort. (C) Performance of IgG Gal-ratio in the detection of individual cancer types in the validation cohort. (D) Performance of IgG Gal-ratio and classical biomarkers for the diagnosis of gastric, liver, lung, ovarian, colorectal, esophageal, pancreatic and prostate cancers in the validation cohort. (E) The positive rates of IgG Gal-ratio marker and classical biomarkers in cancer patients, and the Gal-ratio positive rates in patients with different classical biomarker statuses in the validation cohort. (F) ROC curve for IgG Gal-ratio to distinguish patients with early-stage cancers from non-cancer controls in the validation cohort. (G) Comparison of Gal-ratio values in serum samples obtained from patients with ovarian cancer and those with inflammatory gynecological diseases (a). ROC curve for lgG Gal-ratio to distinguish patients with ovarian cancer from those with inflammatory gynecological diseases (b).

healthy controls, and 272 individuals with non-malignant diseases including chronic hepatitis B (CHB), chronic inflammation and benign tumors. Clinicopathological characteristics of all participants were summarized in Supplementary information, Table S1A and S1B. Consistently, cancer patients showed higher Gal-ratios (median
0.6498, IQR 0.4859-0.8993, mean 0.7482, SD 0.006120) compared with non-cancer controls (median 0.3542, IQR 0.2789-0.4369; mean 0.3784, SD 0.004978; $P<0.0001$ ) in the validation cohort (Figure 1A).

Next the ROC curve was used to evaluate the performance of IgG Gal-ratio in cancer diagnosis. AUC of the 
Gal-ratio is 0.871 ( $95 \%$ confidence interval (CI): 0.879 , 0.903 ) with sensitivity of $79.0 \%$ and specificity of $85.1 \%$ in the test cohort (Supplementary information, Figure S1C and Table S1E), and is 0.862 (95\% CI: $0.712,0.988)$ with sensitivity of $77.8 \%$ and specificity of $83.0 \%$ in the validation cohort (Figure 1B and Supplementary information, Table S1F). Similar result was obtained (AUC $=0.887$ ) when comparing patients with cancer to those with non-malignant diseases. Participants were then classified into three subgroups based on age, each with a 1:1 male-to-female ratio. Performance of IgG Gal-ratio in three subgroups was further evaluated. Although the age, to some extent, has an influence on the values of Gal-ratio, the Gal-ratios of cancer patients were still consistently higher than those of controls in each subgroup (Supplementary information, Table S1D), indicating that the influence of age on IgG galactosylation is unlikely to cause the considerable difference in the Gal-ratios between cancer patients and controls.

Meanwhile, we assessed the diagnostic performance of IgG Gal-ratio analysis in individual cancer. As compared with the controls, the Gal-ratios were found to be consistently significantly higher in each cancer type. ROC analyses demonstrated that the Gal-ratio yielded high AUCs for each of the 12 cancer types, ranging from 0.688 to 0.942 , in the test cohort (Supplementary information, Figure S1D and Table S1E). Notably, the AUCs were $>0.9$ in seven types of cancer, including cancers with the highest mortality in China (lung, liver, esophageal and colorectal cancers). Thus, the Gal-ratio of total serum IgG exhibited a common feature among all the 12 types of cancer and showed great potential in cancer diagnosis. In the validation cohort, the Gal-ratio showed similar performance to that achieved in the test cohort, with AUCs of 0.667-0.930 for the 12 types of cancer (Figure 1C and Supplementary information, Table S1F), verifying that the IgG Gal-ratio exhibited a common feature across the 12 types of cancer and thus represents a promising pan-cancer biomarker. Additionally, the false positive rate and positive and negative predictive values of the Gal-ratio in the test and validation cohorts for each cancer type were listed in Supplementary information, Table S1G.

Considering the accuracy of diagnosis, IgG Gal-ratio showed better performance than classical biomarkers. In the test cohort, the Gal-ratio had greater AUC and higher sensitivity at the same specificity than all classical biomarkers when detecting the corresponding individual cancer. For example, AUCs were 0.942 vs 0.750 and sensitivities were $84.0 \%$ vs $61.2 \%$ at the same specificity for the Gal-ratio vs AFP in detection of liver cancer (Supplementary information, Figure S1E and Table S1E). The performance was further improved when Gal-ratio and classical biomarkers were combined (Supplementary information, Figure S1E and Table S1E). These findings were also verified in the validation cohort (Figure 1D and Supplementary information, Table S1F).

Of note, the positive rate of the Gal-ratio biomarker is higher than that of classical biomarkers in individual cancer type in the test cohort (Supplementary information, Figure S1F). For example, the positive rates of Gal-ratio and AFP in liver cancer are $84.0 \%$ and $58.0 \%$, respectively. Additionally, most classical biomarker-negative cases of cancer were positive for the Gal-ratio, such as Gal-ratio positive rates of $85.7 \%, 77.3 \%, 90.0 \%$ for AFP-, CEA- and PSA-negative cases, respectively (Supplementary information, Figure S1F). The positive rates for the Gal-ratio marker were similar between classical biomarker-positive and -negative patients (Supplementary information, Figure S1F). These data suggest that the diagnosis potential of the Gal-ratio marker is independent of classical biomarker statuses. In addition, the validation cohort confirmed the potential of Gal-ratio as a cancer biomarker independent of classical biomarker statuses (Figure 1E).

Furthermore, we evaluated whether the IgG Gal-ratio holds potential to identify early-stage cancers. In the present study, tumors of stage I were taken as early-stage cancers. The Gal-ratios were significantly different between stage I cancer patients $(n=113)$ and controls in the test cohort. The AUC of Gal-ratio was $0.864(95 \% \mathrm{CI}$ : $0.817-0.910$ ) with the sensitivity of $78.8 \%$ and specificity of $85.1 \%$ (Supplementary information, Figure S1G and Table S1E). When comparing stage I patients $(n=$ 511) and controls in the validation cohort, we found that the Gal-ratio had similar diagnostic accuracy to that in the test cohort with an AUC of 0.848 (95\% CI: 0.8270.868 ) with the sensitivity of $75.0 \%$ and specificity of $82.5 \%$ (Figure 1F and Supplementary information, Table S1F). Similarly, the AUC of Gal-ratio was 0.857 (95\% CI: $0.834-0.880$ ) with the sensitivity of $76.3 \%$ and the specificity of $82.5 \%$ when cancer patients who had only a tumor of $\leq 2 \mathrm{~cm}$ compared to controls in the validation cohort. Thus, Gal-ratio may also be used for the diagnosis of early-stage cancers.

In addition, we assessed whether IgG Gal-ratio can distinguish between cancer and inflammation diseases. Previous reports have provided evidence linking ovarian cancer with some inflammatory gynecological diseases, such as pelvic inflammatory disease, endometriosis and polycystic ovary syndrome [9]. CA125, a routinely used cancer biomarker for ovarian cancer, is usually also elevated in these inflammatory diseases, leading to high incidence of false-positive results. Here, we found that the IgG Gal-ratios were significantly higher in patients with ovarian cancer (median 0.7360, IQR 0.5274-1.062, 
mean 0.8765 , SD 0.02638) compared with those with inflammatory gynecological diseases (median 0.3236 , IQR 0.2603-0.3932, mean 0.3594, SD 0.01592; $P<$ 0.0001 ) (Figure $1 \mathrm{Ga}$ ). ROC analysis yielded AUC of 0.916 (95\% CI: $0.884,0.949)$ for the IgG Gal-ratio (Figure $1 \mathrm{~Gb})$. These results indicate a great performance of $\mathrm{IgG}$ Gal-ratio in discrimination between patients with ovarian cancer and those with inflammatory gynecological diseases. Similarly, compared with CHB $(n=35$; median 0.4480, IQR 0.3306-0.6191, mean 0.4831, SD 0.03644), the Gal-ratios were significantly higher in liver cancer $(n$ $=521$; median 0.7648, IQR 0.5885-1.100, mean 0.9172, SD $0.02390 ; P<0.0001$ ) (Supplementary information, Figure S2A). IgG Gal-ratio showed accurate results in distinguishing patients with liver cancer from those with CHB (AUC 0.833 (95\% CI: 0.765-0.901); Supplementary information, Figure S2A). Similar performance of IgG Gal-ratio was also observed between patients with gastric cancer $(n=1049)$ and patients with gastric inflammation diseases $(n=11)$. IgG Gal-ratio had an AUC of 0.873 (95\% CI: 0.797, 0.949; Supplementary information, Figure S2B). The diagnostic potential of IgG Gal-ratio in discrimination between other types of cancer and inflammatory diseases needs to be further investigated in the future.

Change of $\operatorname{IgG}$ galactosylation was first reported in rheumatoid arthritis [3] and later also reported in other autoimmune diseases, such as psoriatic arthritis and ankylosing spondylitis [10]. Most of autoimmune diseases have their own characteristic symptoms and diagnostic methods, enabling discrimination from cancers. However, it is interesting that similar alterations of IgG glycosylation were observed in both autoimmune disease and cancer. Some studies have suggested possible mechanism for the change of IgG galactosylation in these diseases, such as downregulated galactosyltransferase activity in plasma cells [11] or host-defense response to the presence of the tumor [6]. The potential role of changes of IgG galactosylation in cancer development and progression is worthy of further in-depth investigation.

In summary, we report for the first time that the Gal-ratio of $\operatorname{IgG}$ exhibits a common feature in multiple cancer types in two independent cohorts by assessing distribution patterns of $\operatorname{IgG}$ galactosylation in 12 types of cancers using large-size samples collected from multiple hospitals. Analysis of IgG Gal-ratio could distinguish these 12 types of cancers from non-cancer controls. Similar result was obtained when IgG Gal-ratio was used to distinguish early-stage cancers from non-cancer controls. All the results indicate that the distribution of $\operatorname{IgG}$ galactosylation has great potential to be used as a non-invasive pan-cancer biomarker for early-stage cancer detection and cancer screening.

\section{Acknowledgments}

We thank Xuefei Wang, Yisheng Wang, Junjie Zhao and Li Liu for sample support and helpful suggestions. This work was supported by the Hi-Tech Research and Development Program of China (863 Program; 2012AA020203), the National Basic Research Program of China (973 Program; 2012CB8221004), the National Key Research and Development Program (2016YFA0501303), the National Basic Research Program of China (973 Program; 2013CB910503), the National Natural Science Foundation (31100586 and 81572352) and Zhixianbiotech.

Shifang Ren ${ }^{1, *}$, Zejian Zhang ${ }^{1,{ }^{*}}$, Congjian $\mathrm{Xu}^{2}$, Lin $\mathrm{Guo}^{3}$, Renquan $\mathrm{Lu}^{3}$, Yihong Sun ${ }^{4}$, Jianming Guo ${ }^{5}$, Ruihuan Qin ${ }^{1}$, Wenjun Qin ${ }^{1}$, Jianxin $\mathrm{Gu}^{1}$

${ }^{I}$ Department of Biochemistry and Molecular Biology, Key Laboratory of Glycoconjugate Research Ministry of Public Health, School of Basic Medical Sciences, Fudan University, Shanghai 200032, China; ${ }^{2}$ Obstetrics and Gynecology Hospital, Fudan University, Shanghai 200032, China; ${ }^{3}$ Department of Clinical Laboratory, Shanghai Cancer Center, Fudan University, Shanghai 200032, China; ${ }^{4}$ Department of General Surgery, Zhongshan Hospital, Fudan University, Shanghai 200032, China, ${ }^{5}$ Department of Urology, Zhongshan Hospital, Fudan University, Shanghai 200032, China

*These two authors contributed equally to this work.

Correspondence: Jianxin $\mathrm{Gu}$

Tel: +86-021-5423-7704; Fax: +86-021-6443-7203

E-mail: jxgu@shmu.edu.cn

\section{References}

1 Polanski M, Anderson NL. Biomark Insights 2007; 1:1-48.

2 Tuccillo FM, de Laurentiis A, Palmieri C, et al. Biomed Res Int 2014; 2014:742831.

3 Parekh RB, Dwek RA, Sutton BJ, et al. Nature 1985; 316:452-457.

4 Kodar K, Stadlmann J, Klaamas K, et al. Glycoconj J 2012; 29:57-66.

5 Yi CH, Weng HL, Zhou FG, et al. Oncoimmunology 2015; 4:e1011503.

6 Bones J, Byrne JC, O'Donoghue N, et al. J Proteome Res 2011; 10:1246-1265.

7 Qian Y, Wang Y, Zhang X, et al. J Proteome Res 2013; 12:4046-4055.

8 Kanoh Y, Mashiko T, Danbara M, et al. Anticancer Res 2004; 24:31353139.

9 Kisielewski R, Tolwinska A, Mazurek A, et al. Ginekol Pol 2013; 84:293-297.

10 Martin K, Talukder R, Hay FC, et al. J Rheumatol 2001; 28:15311536.

11 Axford JS, Sumar N, Alavi A, et al. J Clin Invest 1992; 89:1021-1031.

(Supplementary information is linked to the online version of the paper on the Cell Research website.)

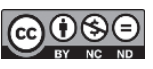

This work is licensed under a Creative Commons Attribution-NonCommercial-NoDerivs 4.0 Unported License. The images or other third party material in this article are included in the article's Creative Commons license, unless indicated otherwise in the credit line; if the material is not included under the Creative Commons license, users will need to obtain permission from the license holder to reproduce the material. To view a copy of this license, visit http://creativecommons.org/licenses/by-nc-nd/4.0/

(C) The Author(s) 2016 